\title{
ON THE RELATION BETWEEN DR. KOETTSTORFER'S PROCESS OF BUTTER ASSAY, AND THE OLD PROCESSES.
}

\author{
By G. W. Wigner, F.C.S.
}

Read before the Society of Public Analysts, at Sheffield, on 22nd August, 1879.

Dr. Koetrstorfer's process appeared to me likely to prove very valuable in the ordinary analyses of butter, by at least disposing without further labour of most of the genuine samples; but it was obviously impossible to adopt a new process as a basis of an adverse report, until it had been thoroughly tested. It appeared to me, that the most practical mode of testing it was to submit to this process (in addition to the tests which would otherwise have been made) every sample of butter or other fat which was received. I therefore adopted it as an auxiliary process, and have examined nearly 100 samples by it.

As a general opinion, I may state that I am well pleased with the process for the purpose above mentioned. It is not, however, free from its weak points.

Carbonic acid acts very much on the colour of phenol-phthalein, and, in order to cnsure fair accuracy, it is essential that the fat should be saponified with the alcoholic potash solution in a flask, which certainly ought to be corked with a cork having a notch in the side. While the alcohol itself is boiling pretty freely there is, of course, no risk of carbonation, but, as soon as the water is added to dilute the soap for titration, the risk increases greatly.

My experience also indicates the necessity for the use of a very large excess of potash solution, an excess very much larger than would be ordinarily employed when the fatty acids were to be weighed. It appears desirable to use at least twice as much potash as would be actually required to combine with the soap present.

Again, in reference to the potash solution itself. I find that a very small amount of colour seriously masks the end of the titration, and it becomes essential to use pure potash, and to keep the solution in hard glass bottles, so as to prevent the additional colour which is often imparted by the decomposition of the glass of common Winchesters.

Taking all these precautions, however, I find the process a useful one. But I must call special attention to the following exceptions:-It is comparatively useless when applied to old samples of butter, which have been alternately heated, and cooled; and, even in the cases of lard and butterine, repeated heating exercises a more uncertain effect than it does on the fatty acid determination; but, although useful, it can never come into general use as a substitute for the determinations of fatty acids and soluble acids, because any alkalies added to the fat, whether frandulently, or for supposei preservative purposes, entirely upset the estimation. Therefore, while it may be-and in my opinion is, when properly carried out-a safe process on which to pass a butter as genuine, it is quite unreliable as a proof that the butter is adulterated. The admixture of three per cent. of carbonate of soda with the salt added to the butter, will, by this process, change the results so much, that a genuine butter would bo condemned; and such a percentage of admixture is one that has been used, while smaller percentages are common. 
By the fatty acids' process, such an admixture would have scarcely any sensible effect, but it would exercise a very important effect on the actual density of the fat, and, indeed, in certain cases, might render the determination of actual density quite fallacious. Unfortunately merely testing the water present in the sample of butter for alkalinity is not sufficient to prove whether alkaline carbonates have been added, and no test seems readily available. I have, therefore, come to the conclusion that it is unsafe and unjust to condemn any sample of butter as adulterated on either the titration or specific gravity processes. If the sample passes these tests well it may be relied on as genuine, but it may fail to answer both tests, and yet have no foreign fat mixed with it. In such a case, a full determination of both soluble and insoluble fatty acids, is the only safe course to adopt.

Of course, therefore, I view the relation between the actual density, fatty acids and titration results as a matter of no great importance in adulterated samples, but in genuine samples it is otherwise.

Dr. Koettstorfer found that the amount of KHO required by genuine butters ranged from $23 \cdot 24$ to $22 \cdot 15$ per cent. It is, of course, with the lowest limits only that we have to concern ourselves, and my opinion is, that this limit is not low enough even in the case of fresh samples, and that it is very seriously deficient in the case of samples which have been kept and subjected to cven moderate variations of temperature.

I have lad several samples of butter apparently recently made, and, certainly, in good condition, which have required as little as $21 \cdot 34,21 \cdot 36,21.50$ per cent. of KHO to saponify them, and which have yet given less than 89 per cent. of fatty acids by the flask washing process, and which, independently of the other conditions, I certainly would not condemn as adulterated. In my opinion, therefore, the titration process can only be relied on when it shows figures higher than Koettstorfer has put as the limits.

As, however, three samples out of every four received are probably genuine, the process may be advantageously used for passing these pure samples, provided proper care is taken to carry the analysis further if these results are adverse, and it is for this purpose only that the process can come into general use.

I think that any sample of butter fat, which requires more than 22.60 per cent. KHO for saponification, as determined by the titration process, may safely be passed as genuine; but that any lower result should be checked by a full analysis. 